04

\title{
Возможность использования отрицательной спиновой компоненты в нейтронных поляризующих устройствах. Первые результаты
}

\author{
(С) Г.П. Гордеев, В.Н. Забенкин, Г.П. Диденко, Л.А. Аксельрод, И.М. Лазебник, В.А. Лямкин \\ Петербургский институт ядерной физики им. Б.П. Константинова научного центра Курчатовский Институт, \\ 188300 Гатчина, Ленинградская обл., Россия \\ T e-mail: lyamkin_va@pnpi.nrcki.ru
}

Поступило в Редакцию 1 июня 2018 г.

В окончательной редакции 4 декабря 2018 r.

Принято к публикации 14 декабря 2018 г.

\begin{abstract}
Приведены результаты экспериментальной проверки возможности повышения светосилы поляризующих нейтронные пучки устройств на базе намагниченных суперзеркал. Суть заключается в использовании обеих компонент спинов ансамбля нейтронов в пучке. Положительная компонента отражается от одного намагниченного зеркала, а проходящая зеркало отрицательная после поворота на $180^{\circ}$ отражается от другого аналогичного зеркала. Оба пучка регистрировались одним детектором. С учетом коэффициентов отражения компонент зеркалами и эффективности поворота отрицательной компоненты получено увеличение интенсивности нейтронов примерно в два раза при средней поляризации около 66\%. Эксперименты выполнены на монохроматическом пучке нейтронов.
\end{abstract}

DOI: 10.21883/JTF.2019.06.47633.216-18

\section{Введение}

В практике получения пучков поляризованных нейтронов обычно используется только одна спиновая компонента (проекция) из ансамбля нейтронов, совпадающая по направлению с характерным направлением в материале, используемом в качестве поляризующей среды (например, с вектором магнитной индукции или вектором поляризации среды). В любом из существующих на сегодняшний день способов получения другая компонента (противоположная первой) не используется вообще. Более того, от нее избавляются посредством поглощения нейтронов этой спиновой компоненты элементами с большим сечением поглощения. Так, в современных поляризаторах на основе отражения нейтронов от намагниченных зеркал/суперзеркал они поглощаются слоем сплава TiGd, нанесенного на ту или иную подложку [1]. В методе получения поляризованных нейтронов с помощью поляризованных мишеней $\mathrm{He}^{3}$ эта компонента автоматически поглощается атомами $\mathrm{He}^{3}$ [2,3]. При большой поляризующей способности таких устройств им присущ существенный недостаток, заключающийся в потере интенсивности (примерно в 2 раза) нейтронных пучков из реактора.

Всегда стоящая задача повышения интенсивности поляризованных нейтронов приобретает первостепенное значение при эксплуатации маломощных пучков (как, например, в зоне бывшей тепловой колонны реактоpa BBP-M).

\section{1. Эксперимент и результаты}

Наиболее простым решением задачи, как нам кажется, является использование второй компоненты, которая те- ряется в современных устройствах получения поляризованных нейтронных пучков. Впервые это решение было предложено в работе Н.К. Плешанова [4], в которой предполагается для этого использовать отражение от специальных зеркал со сложным многослойным напылением, способным отражать нейтроны с перевернутой поляризацией (флиппер-зеркало). Для этого современные суперзеркала нужно изготавливать напылением отражающих магнитных слоев (например, тех же чередующихся пар слоев $\mathrm{CoFe} / \mathrm{TiZr}$ [1]) на слабопоглощающую подложку из $\mathrm{Si}$ без поглощающего слоя $\mathrm{TiGd}$. Спиновая компонента, совпадающая с направлением намагничивающего поля, в этом случае отражается, а противоположная ей - проходит через зеркало и кремний с неизбежным преломлением пучка прошедших нейтронов. Если спины этих нейтронов повернуть на $180^{\circ}$ и направить пучок на следующее аналогичное зеркало, находящееся на некотором расстоянии от первого в магнитном поле того же направления, то нейтроны этой компоненты отразятся от него. Отраженные пучки нейтронов с одним направлением спинов регистрируются общим детектором (в нашем случае двухкоординатным детектором с размером ячейки $x=y=0.6 \mathrm{~mm}$, называемой каналом детектора). Приведенные ниже результаты измерений представляют зависимости суммы нейтронных импульсов в $X$-ячейках при данном направлении по $Y$ от номера $Y$-ячейки (канала), названные нами на рисунках ниже интенсивностью в произвольных единицах.

Эксперимент осуществлялся на рефлектометре монохроматических поляризованных нейтронов „Реверанс“ $[5,6]$ с длиной волны нейтронов $\lambda=0.54 \mathrm{~nm}$ и с немонохроматичностью $\Delta \lambda / \lambda \approx 2 \%$. На рис. 1 представлена схема эксперимента, которая включала в себя следующие узлы: шим для создания неполяризованно- 


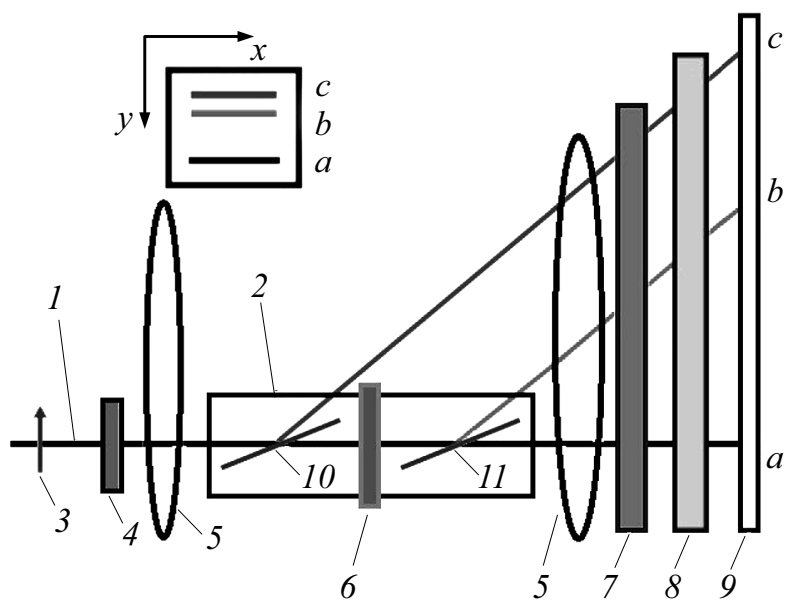

Рис. 1. Схема эксперимента: 1 - поляризованный пучок нейтронов, 2 - кассетница, 3 - направление поляризации, 4 - шим, 5 - катушки для создания горизонтального ведущего поля, 6 - пленочный магнит, 7 - флиппер $\mathrm{F}_{2}, 8-$ анализатор поляризации, 9 - двухкоординатный детектор, 10 - суперзеркало № 1, 11 - суперзеркало № 2. На вставке: на экране $2 D$-детектора с координатными осями показаны проходящий пучок $(a)$, отраженный от зеркала № $2(b)$ и отраженный от зеркала № 1 (c).

го пучка, пару катушек для создания горизонтального ведущего поля, кассетницу с зеркалами, пленочный магнит между зеркалами, флиппер, анализатор и двухкоординатный детектор нейтронов. Для определения эффективности входящих в схему узлов (шим и пленочный магнит), а также для определения поляризации исходного пучка использовались 2 флиппера: флиппер $\mathrm{F}_{1}$ перед зеркалами (он не показан на рисунке, так как стоит далеко перед шимом) и флиппер $\mathrm{F}_{2}$ после них. На деполяризованном пучке использовался только флиппер $\mathrm{F}_{2}$. Форма исходного поляризованного пучка нейтронов показана на рис. 2 при четырех состояниях флипперов. Средняя поляризация пучка составляла 0.86 .

Шим - лист жести - полностью деполяризовал этот пучок (рис. 3). Интенсивности нейтронов в „темной“ и в „светлой“ позициях флипперов ( $I_{D}$ и $I_{L}$, соответственно) практически равны, и поэтому поляризация $P=\left(I_{L}-I_{D}\right) /\left(I_{L}+I_{D}\right) \approx 0$. В специально изготовленную кассетницу помещались обоймы с изготовленными суперзеркалами на кремниевой подложке без поглощающего слоя TiGd. Пластины $\mathrm{Si}$ размером $100 \times 37 \times 0.47 \mathrm{~mm}$, на полированную сторону которых напылялись суперзеркальные покрытия по принятой у нас технологии (68 пар слоев CoFe/TiZr с переменным периодом), имели тыльную сторону лишь шлифованную, т.е. сильно шероховатую. Согласно технологии изготовления, зеркала должны были иметь остаточную индукцию до $5 \mathrm{mT}$ [7-9]. Из таких реманентных зеркал уже давно изготавливают нейтронные поляризаторы и анализаторы [10,11]. Зеркала располагались так, чтобы неотраженный пучок нейтронов от первого зеркала проходил его и падал на поверхность второго зеркала. Нейтронный пучок падал на зеркало под углом, близким к критическому, для указанной длины волны нейтронов, равным $18 \mathrm{mrad}$. В кассетнице было отверстие для помещения пленочного магнита, представляющего собой пленку из сплава $\mathrm{SmCo}_{5}$ толщиной $d=28 \mu \mathrm{m}$, нанесенную на титановую пластину размером $80 \times 250 \mathrm{~mm}$ и толщиной $L=0.5 \mathrm{~mm}$. Остаточная индукция такой пленки $B=4500 \mathrm{G}$, коэрцитивная сила $H_{C}=3500$ Ое. При таких параметрах и $\lambda=0.54 \mathrm{~nm}$ поляризация $P$ при нормальном падении нейтронного пучка на магнит должна испытывать поворот на угол $\Theta=C B d \lambda=\pi \mathrm{rad}$, где константа $C=4.63 \cdot 10^{14} \mathrm{~T}^{-1} \cdot \mathrm{m}^{-2}$. Это и видно из рис. 4. Максимальная интенсивность нейтронов достигается в состояниях, когда $\mathrm{F}_{1}$ включен (1), а $\mathrm{F}_{2}$ выключен (0) и когда $\mathrm{F}_{1}$ выключен $(0)$, а $\mathrm{F}_{2}$ включен (1), т.е. когда 1-й и 2-й флипперы включены (темная позиция). В состояниях, когда оба флиппера выключены (состояние 00) или включены (состояние 11), интенсивность

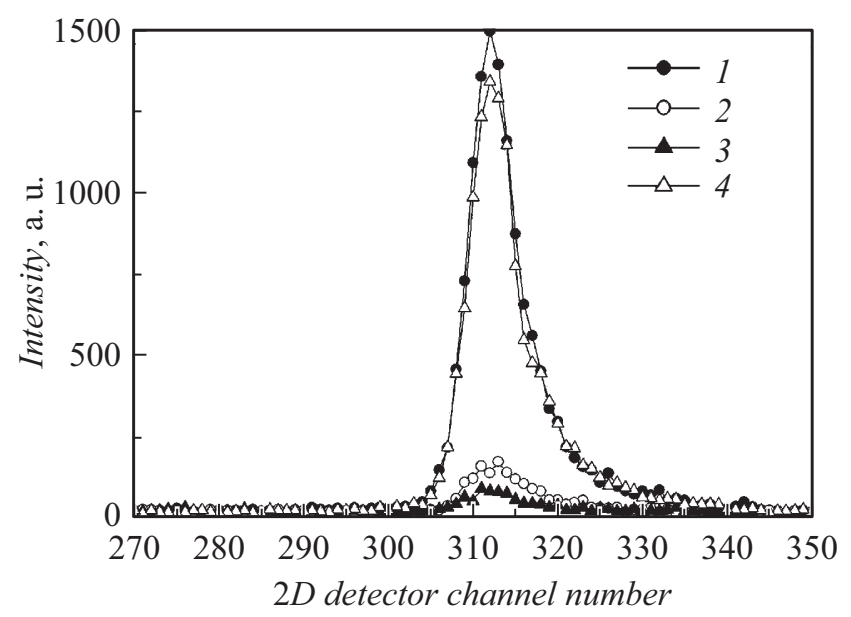

Рис. 2. Спектр поляризованного исходного пучка при четырех состояниях флипперов: $1-00,2-10,3-01,4-11$. Первый индекс в комбинации 0 и 1 относится к флипперу $\mathrm{F}_{1}$, второй - к $\mathrm{F}_{2} .0-$ флиппер выключен, 1 - флиппер включен.

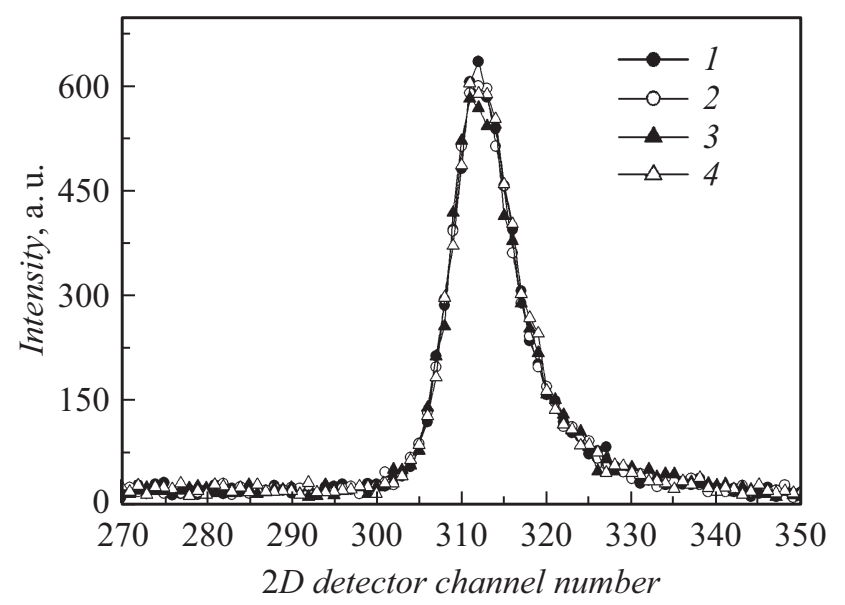

Рис. 3. Спектр деполяризованного шимом пучка при четырех состояниях флипперов: $1-00,2-10,3-01,4-11$. 


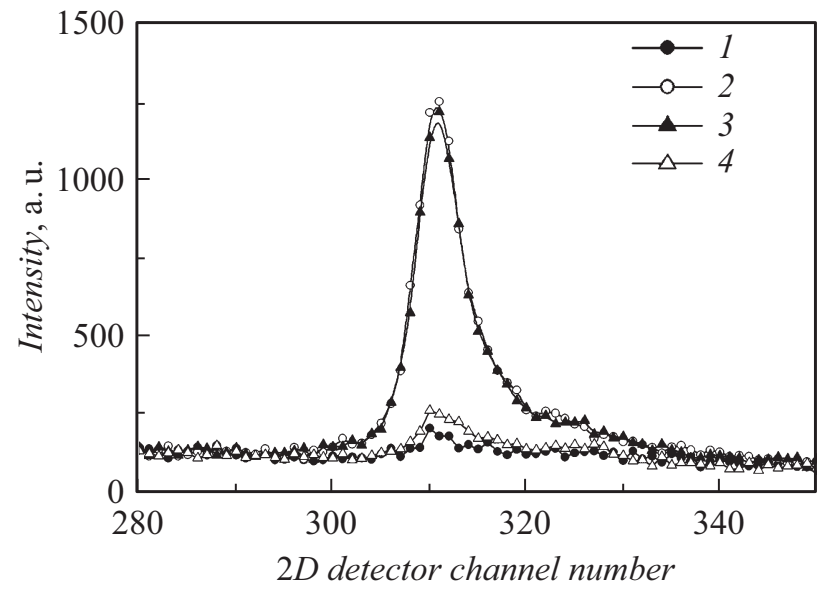

Pис. 4. Спектр поляризованного пучка нейтронов после прохождения пленочного магнита в четырех состояниях флипперов: $1-00,2-10,3-01,4-11$.

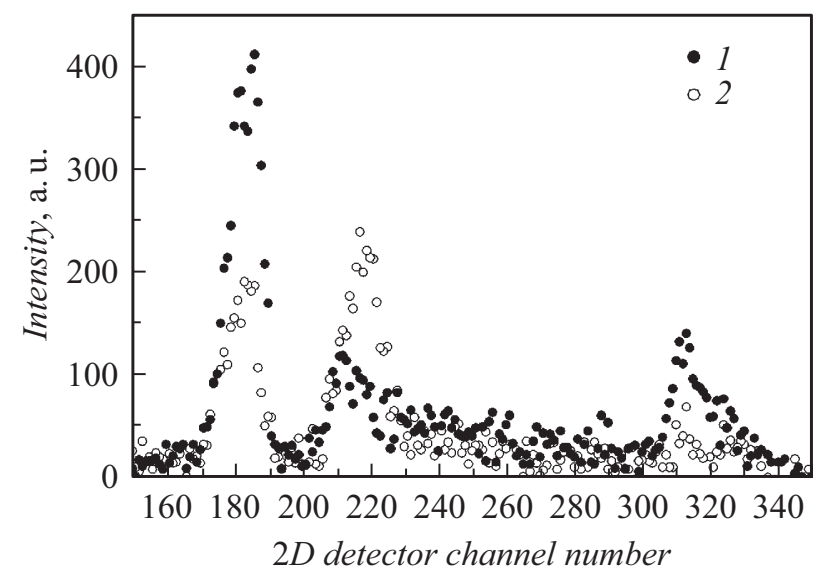

Рис. 5. Отраженные пучки от зеркал № 1 и № 2 в двух состояниях флиппера $\mathrm{F}_{2}(1-00,2-01)$ на неполяризованном пучке. Магнит отсутствует.

минимальна. При сравнении рис. 2 и рис. 4 видно, что максимальные интенсивности наблюдаются при противоположных состояниях флипперов, а вычисленная по приведенной выше формуле средняя поляризация в диапазоне 310-325 канала детектора (рис. 4) по флипперу $\mathrm{F}_{1}$ равна $P \approx-0.854$, т. е. происходит поворот приблизительно на $180^{\circ}$, точнее на $174^{\circ}$. По второму флипперу поляризация равна $P \approx-0.75$, вследствие меньшей эффективности переворота поляризации. При этом угол поворота оказался равным $\Theta \approx 151^{\circ}$. Предварительные измерения коэффициентов отражения каждого зеркала проводились на поляризованном пучке как для положительной $\left(R^{+}\right)$, так и отрицательной $\left(R^{-}\right)$ компонент. Для первого (входящего) зеркала $R^{+}=0.60$, $R^{-}=0.66$, а для второго эти отражения 0.48 и 0.53 соответственно. Уже эти значения свидетельствуют об индукции магнитных покрытий зеркал, далекой от насыщения.
Поляризующая способность зеркал в такой сборке проверялась на пучке с шимом, т.е. на неполяризованном пучке. Измерение поляризации проводилось с помощью флиппера $F_{2}$. Пики отраженных от зеркал нейтронов в двух состояниях флиппера расположены в районе 180 и 220 каналов. В районе 315 канала находится остаток прошедшего пучка. Из рис. 5 видно, что оба отраженных пучка демонстрируют некоторую поляризующую способность зеркал. Средняя поляризация пучка, отраженного первым зеркалом, составляет $P_{1}=0.60$, а для отраженного от второго $P_{2}=-0.45$. Это находится в соответствии с коэффициентами отражения компонент. Видно также, что компоненты разного знака отражаются в разные углы в соответствии с тем, что показатель преломления зависит от направления спина нейтрона относительно индукции зеркал. При установке пленочного магнита между зеркалами картина существенно меняется (рис. 6). Во-первых, поляризации обоих отраженных пучков имеют один знак. Во-вторых, практически при неизменной средней поляризации первого отраженного пучка $\left(P_{1} \approx 0.61\right)$ средняя поляризация второго $P_{2}$ возросла до $\sim 0.71$, так что суммарная средняя поляризация составляет примерно 0.66 . В-третьих, отношение интенсивностей обоих отражений близко к единице (точнее $I_{1} / I_{2} \approx 0.96$ ).

При идеальных условиях (не меняющих проводки поляризации) и при использовании двумерного детектора, два поляризованных пучка, находящихся на некотором расстоянии друг от друга, можно свести в одну точку изменением углов отражения зеркал (в области до критического) и расстояния между ними. Равенство интенсивностей пиков свидетельствует о малом ослаблении нейтронных пучков кремниевыми подложками зеркал и пленочным магнитом. Суммарная средняя поляризация пиков так же, как и поляризации каждого в отдельности, сильно отличается от поляризации первичного (рис. 3) пучка $P=0.86$.

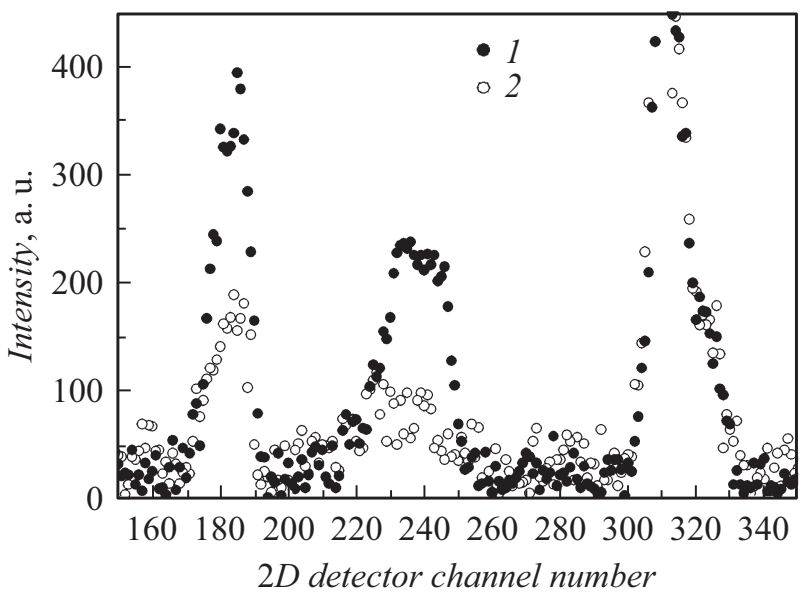

Рис. 6. То же, что на рис. 5 , но в присутствии пленочного магнита между зеркалами. Направления поляризации в отраженных пиках совпадают. Магнит повернул отрицательную спиновую компоненту, прошедшую от зеркала № 1. 


\section{2. Обсуждение результатов}

Разумеется, это отличие связано как с поляризационными характеристиками зеркал, так и с влиянием рассеянных магнитных полей от пленочного магнита, а также с недостаточной резкостью неадиабатических границ в районе пленочного магнита. Конечно, основным определяющим узлом в этой схеме является магнитная система для поворота отрицательной спиновой компоненты на $180^{\circ}$. Магнитное поле ее имеет направление, перпендикулярное полю зеркал, что затрудняет создание строго неадиабатической области между зеркалами. Эта проблема сильно усложняет реализацию идеи на практике. Наиболее приемлемым приближением к решению проблемы увеличения светосилы поляризующих устройств является использование зеркал с большой остаточной индукцией и достаточной высокой коэрцитивной силой, а в качестве вращателя отрицательно поляризованного пучка - электромагнита типа тороида. Он не создает рассеянных магнитных полей, поэтому условия проведения поляризации не изменятся при помещении его в пространство между зеркалами. Мысль об устройстве, использующем это свойство тороида, пришла к нам поздно, когда реактор останавливался надолго.

Таким образом, можно сказать, что идея повышения светосилы поляризующих устройств для монохроматических нейтронов подтверждается. Что касается практического использования, то предпочтительно использование зеркал с большой реманентностью. В этом случае, возможно, удастся обойтись вообще без магнитной системы, если намагниченности зеркал направлять в противоположные стороны. Правда, придется как-то адиабатически вывести поляризацию во втором отраженном пучке на поле анализатора. В спешном порядке мы попытались провести подобный эксперимент (рис. 7).

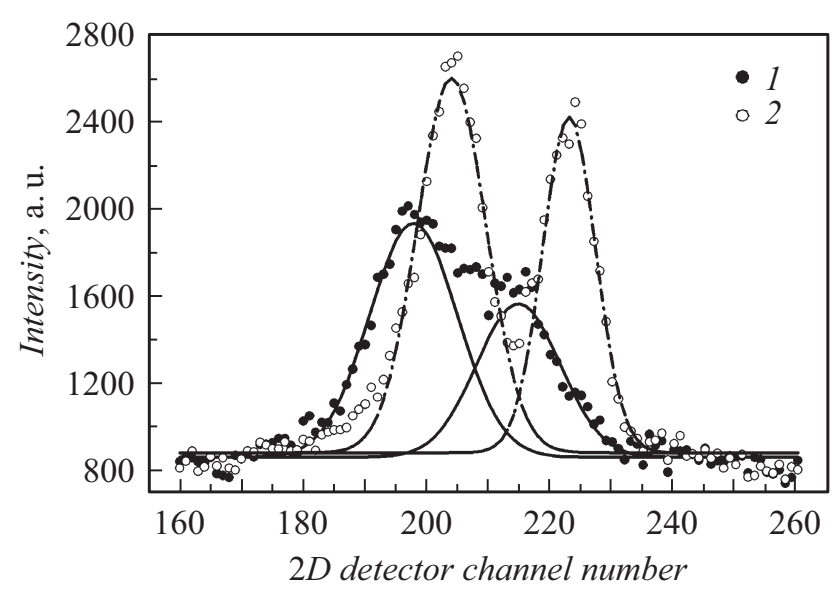

Рис. 7. Отраженные пучки от зеркал № 1 и № 2 с противоположными направлениями остаточных индукций при двух состояниях флиппера $\mathrm{F}_{2}(1-00,2-01)$. Линии (сплошная и штрихпунктирная) представляют собой результат разложения экспериментальных данных при подгонке. Прошедший пучок не показан.

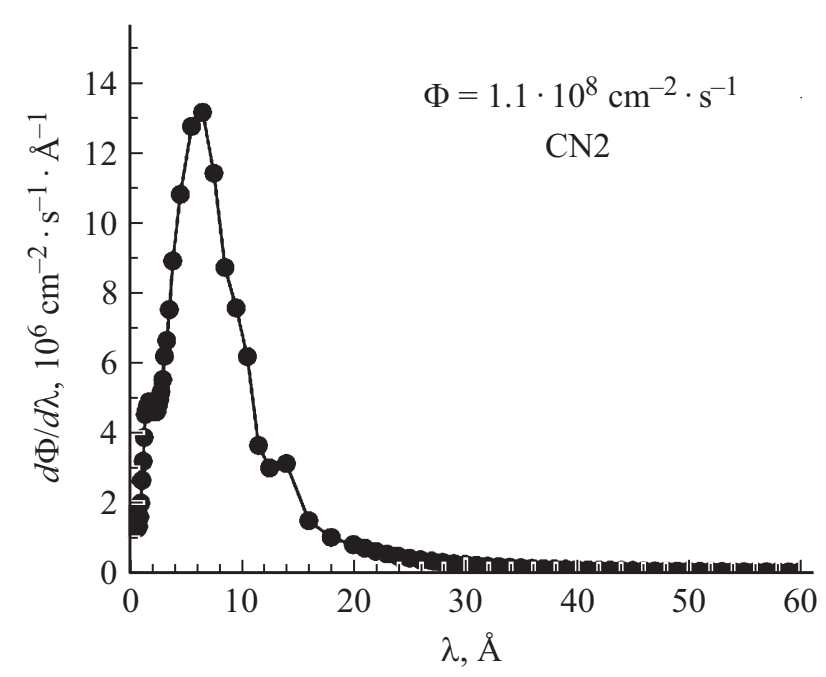

Рис. 8. Плотность нейтронного потока $d \Phi / d \lambda$ на выходе прямого нейтроновода ХН2.

Поведение интенсивностей отражений в „темной“ позиции кажется удовлетворительным, а в „светлой“ отрицательные компоненты поляризации отраженных пучков не разрешаются. При работе с двумерным детектором это не особенно важно, поскольку отражения $c$ и $b$ (рис. 2) интегрируются. Разлагая полученные кривые, имеем среднюю поляризацию около $P \approx 0.15$. Следует заметить, что для широких падающих пучков нейтронов потребуется использование нескольких пар зеркал, организованных в две стопки. Они находятся на определенных расстояниях друг от друга как в продольном направлении, так и по высоте, чтобы предотвратить поглощение отраженных пучков от зеркал первой стопки. Для поворота прошедшей спиновой компоненты удобно использовать либо систему типа „тороид“, либо использовать большую остаточную индукцию зеркал с противоположным направлением в стопках.

На сегодняшний день не все полученные результаты понятны. Во-первых, это - большая ширина второго пика по сравнению с первым и форма самого пика. Вклад в уширение могут дать шероховатости тыльной стороны 1-го зеркала, разный угол поворота поляризации в магните из-за разброса длин волн нейтронов и незеркальное отражение как первым, так и вторым зеркалами Тонкая структура пика наводит на мысль об интерференции двух преломленных пучков (+ и - компонент). Во-вторых, малый коэффициент отражений $R$ для + и - поляризаций. Об этом говорит достаточно интенсивный прошедший пучок. Видимо, к концу эксперимента уменьшилась намагниченность зеркал, поэтому изменились и коэффициенты отражений. По оценкам их значения стали $R_{1}^{+}=0.56, R_{1}^{-}=0.60, R_{2}^{+}=0.44$, $R_{2}^{-}=0.60$. И наконец, большой общий фон. Наиболее вероятно, он связан с некогерентным рассеянием титановой подложки магнита. Форма второго пика, вероятно, зависит от эффективной толщины магнита. При повороте магнита вокруг продольной оси на 15 градусов большее изменение в форме испытывает второй пик. 


\section{Заключение}

В заключение отметим, что к анализу указанных фактов нам еще предстоит вернуться, привлекая весь архив измерений, но, несмотря на это, уже сегодня можно утверждать, что идея, озвученная вначале, подтверждается. По крайней мере, для монохроматического пучка нейтронов. Предстоит рассмотреть и варианты конструкций таких поляризующих устройств.

Что касается измерений с квазимонохроматическим спектром, то нам не хватило времени детально провести эксперимент. Реактор был на длительное время остановлен. Это последняя наша работа на реакторе ВВР-М.

Продолжить работу мы планируем после ввода в эксплуатацию нового источника УХН на сверхтекучем гелии [12]. Источник в настоящее время находится в фазе изготовления. Он будет смонтирован в тепловой колонне реактора ВВР-М внутри графитового блока и будет состоять из камеры предзамедлителя, заполняемой жидким дейтерием, и самой гелиевой камеры, заполненной сверхтекучим гелием. Температура жидкого дейтерия в камере предзамедлителя будет поддерживаться на уровне $20 \mathrm{~K}$. Расчетная плотность потока холодных нейтронов $d \Phi / d \lambda$ на выходе прямого нейтроновода ХН2 $1.1 \cdot 10^{8} \mathrm{~cm}^{-1} \cdot \mathrm{s}^{-1}$ (рис. 8).

\section{Благодарности}

Мы благодарны техническому персоналу реактора BВР-М за добросовестное отношение ко всем нашим просьбам, возникавшим при выполнении данной работы. Мы благодарны сотрудникам „отдела нейтронной оптики“ за изготовление зеркал для данного эксперимента и сотрудникам отдела ОИКС за обсуждение и критические замечания.

\section{Финансирование работы}

Отдельные части данной работы выполнены в НИЦ „Курчатовский институт“ - ПИЯФ за счет гранта Российского научного фонда (проект № 14-22-00105).

\section{Список литературы}

[1] Schebetov A.F., Pleshanov N.K., Pusenkov V.M., Peskov B.G., Shmelev G.E., Kraan W.H., Por T., Rekveldt M.Th., Mikhailova V.E. // Nucl. Instrum. Method. B. 1994. Vol. 94. P. 575.

[2] Tasset F., Chupp T.E., Pique J.P., Stlinhof A., Thompson A., Wasserman E., Ziade M. // Phys. B. 1992. Vol. 180-181. N 2. P. 896-898.

[3] Tasset F., Ressouche E. // Nucl. Instrum. Method. Sect. A. 1995. Vol. 359. P. 537-541.

[4] Pleshanov N.K. // J. Phys.: Conf. Ser. 2014. Vol. 528. P. 012023.

[5] Раджабов А.К., Гордеев Г.П., Аксельрод Л.А., Лазебник И.М., Забенкин В.Н. // Кристаллография. 2007. Т. 52. № 3. С. 585.
[6] Radzhabov A.K., Gordeev G.P., Lazebnik I.M., Axelrod L.A., Zabenkin V.N. // Physica B. Cond. Matter. 2007. Vol. 397. P. 156.

[7] Boni P., Clemens D., Senthie Kumar M., Pappas C. Physica B. 1999. Vol. 267-268. P. 320.

[8] Pleshanov N.K., Bodnarchuk V., Galer R. et al. // Physica B. 2001. Vol. 297. P. 126.

[9] Senthie Kumar M., Boni P. // J. Appl. Phys. 2002. Vol. 91. P. 3750.

[10] Pappas C., Kali G., Krist N. et al. // Physica B. 2000. Vol. 283. P. 365.

[11] Плешанов Н.К., Аксельрод Л.А., Забенкин В.Н., Сыромятников В.Г., Ульянов В.А. // Поверхность. 2008. № 11. C. 3 .

[12] Serebrov A.P. // Crystallography Reports. 2011. Vol. 57. N 7. P. 1230 . 